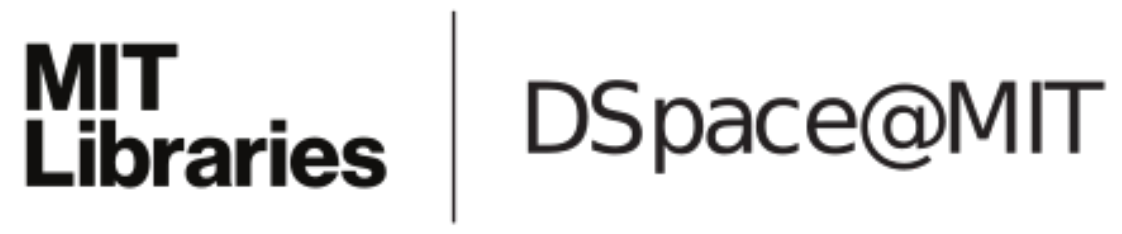

\author{
MIT Open Access Articles
}

How It Is Made Matters: Distinguishing Traits of Designs Created by Sketches, Prototypes, and CAD

The MIT Faculty has made this article openly available. Please share how this access benefits you. Your story matters.

Citation: Tsai, Geoff, and Maria C. Yang. "How It Is Made Matters: Distinguishing Traits of Designs Created by Sketches, Prototypes, and CAD." Volume 7: 29th International Conference on Design Theory and Methodology (August 6, 2017).

As Published: http://dx.doi.org/10.1115/DETC2017-68403

Publisher: ASME International

Persistent URL: http://hdl.handle.net/1721.1/120045

Version: Final published version: final published article, as it appeared in a journal, conference proceedings, or other formally published context

Terms of Use: Article is made available in accordance with the publisher's policy and may be subject to US copyright law. Please refer to the publisher's site for terms of use. 


\section{HOW IT IS MADE MATTERS: DISTINGUISHING TRAITS OF DESIGNS CREATED BY SKETCHES, PROTOTYPES, AND CAD}

\author{
Geoff Tsai \\ Department of Mechanical Engineering \\ Massachusetts Institute of Technology \\ Cambridge, MA 02139
}

\author{
Maria C. Yang \\ Department of Mechanical Engineering \\ Massachusetts Institute of Technology \\ Cambridge, MA 02139
}

\begin{abstract}
In the early stages of design, designers may use a variety of tools to represent their ideas, including sketches, physical prototypes, and digital models. Prior research suggests that the choice of tool and design representation can influence user opinions of the concept. In this paper, we explore how aware designers and users are of the ways different design tools can influence a design. Specifically, we investigate the question "How is a design influenced by the tool used to create it?" Designs that had originally been created as either a sketch, foam prototype, or CAD model were sketched into a consistent visual style. Designers experienced with these tools exhibited a better-than-random likelihood of identifying the original tool used to create the design, despite viewing only the re-sketch. This suggests artifacts of a design tool persist in a design representation despite the design being translated from one medium to another.
\end{abstract}

\section{INTRODUCTION}

Design teams may use a variety of tools to generate and represent provisional designs. These tools can range from paper-based 2D sketches, to digital models, to 3D physical prototypes - each with varying levels of fidelity, cost, and speed to produce. Prior work by Häggman, et al. [1], suggests the choice of tool can influence user perceptions of a design, including its novelty, usability, and aesthetics. As described by Schon [2], the process of designing can be thought of as a "conversation" with the materials while designing, but we ask "can the choice of these materials be observed after the fact?". This paper examines the questions: "How is a design influenced by the tool used to create it? Can designers determine the tools that were used to create a design? And how do their responses relate to the attributes of that design?". For example, a design might be perceived as "blocky" or "delicate" or possessing "symmetry". Furthermore, these visual representation attributes may be linked with specific design tools - a "blocky" design may be a clue that it was created using CAD because of the ease of creating geometric shapes, while a "delicate" design may suggest that the design could not have been created as a foam model because of the difficulties of working with delicate parts in that material. Studying how these tools can influence the design outcome has the potential to enable designers to become more aware of the choices they make when they choose to use a tool. Understanding how stakeholders respond to a design representation is vital to interpreting potential user feedback on a design accurately. This paper investigates the research questions:

Can designers correctly identify the tool used to create a particular design?

What design attributes do designers associate with different design tools?

Our expectation is that more experienced designers will be able to determine design tools more accurately than novices, and that different design tools will be linked to different design attributes.

To evaluate this question, we created a survey that showed re-sketched designs for a product created using sketching, physical prototyping, or CAD and asked design practitioners to identify the tool they believed was used.

\section{RELATED WORK}

\section{Design Tool Comparisons}

There is current research into understanding and comparing the use of different design tools, although there is less research into comparing physical prototypes to other tools. Acuna and Sosa [3], in measuring sketching and physical 
prototyping during a design exercise, observed tradeoffs with time spent during each activity, with greater prototyping time associated with higher-quality models, but greater sketching time associated with higher creativity. Viswanathan and Linsey [4] used different prototyping materials, including sketching, and found that the creation of physical models was linked to a higher percentage of functional ideas. In focusing on the psychological experience of design activity from the perspective of the designer, Gerber and Carroll [5] observed designers creating low-fidelity prototypes. In creating many quick, low-fidelity prototypes, designers were able to view failed ideas as examples of learning, and the set of ideas prototyped as an affirmation of their own creativity.

Much of the focus of design tool comparison research is on paper sketching versus digital tools. Black [6], in a survey of graphic design students, noted that compared to users of paper tools, users of digital tools admitted to compromising some of their initial ideas because of workflows in the software. Bilda and Demirkan [7] studied how interior designers approached a design problem using traditional sketching tools versus 3D CAD tools and noted that users of traditional tools more frequently modified the goals of their solutions, thus CAD tools may be artificially limiting during the conceptual design phase. Vasantha, et al. [8] conducted a controlled study comparing designers using pen-on-paper (with digital recording), drawing tablet, or 3D CAD to solve design problems. Their results found use of different tools had an impact on how long each designer spent solving each problem, as well as the proportion of detailed design activity.

\section{Product Perception}

Product perception deals with how a product's appearance and meaning are interpreted by the user. Many different and inter-related factors can influence product perception, and it is an area of interest for research. In addition to form, color may also have an influence on product perception. Mugge and Schoormans [9] varied the colors of 3D models and observed a positive association between the perceived novelty of a product and its level of performance. Pérez Mata, et al. [10] surveyed how perceptions and aesthetics of product relate to a consumer's desire to own that product, and they developed a set of guidelines for creating a product for a specific effect.

Perception of a design can be divided into two components: the representation fidelity and the representation mode. Where fidelity is concerned with the level of detail and realism of the representation, and mode is concerned with the medium, e.g. sketches, physical models, photographs, 3D software models, etc. Different levels of fidelity can be achieved, for example, with sketches containing more or less detail, or from sketches that range from concrete to abstract. An effect of this range of concrete to abstract is the level of interpretation required by the viewer: from a message "received" (zero interpretation) to a message that is "perceived" [11].

\section{Representation Fidelity}

Prior research has investigated aspects of the connection between the design representation and user preferences. Focusing on the quality of representation within a given representation mode, Kudrowitz, et al. [12] found a link between sketch quality and the perceived creativity of a product idea. Sauer and Sonderegger [13] tested the impact of prototype fidelity - including paper prototype, computer prototype, and fully-working interface - on a usability test, and found the computer prototype (medium-level fidelity) over-estimated the task completion time. Macomber and Yang [14], using sketches with varying levels of fidelity, found that crowdsourced-responders preferred high-fidelity sketches over low-fidelity sketches. Viswanathan and Linsey [15] observed that quick prototypes are associated with less premature fixation, potentially helping designers avoid the sunk cost fallacy by lowering the barrier to switch design directions. Yang [16], studying the performance of mechanisms in an undergraduate course, found that simpler prototypes were associated with a better course grade and design outcome. Hannah, et al. [17] surveyed different modes of representation, although focused on the levels of fidelity of those representations, and found designers can extract more information and are more confident that a design meets requirements when viewing a high-fidelity representation. In addition, low-fidelity representations were faster to create and were effective at representing functional requirements, they did not provide enough information about geometric or manufacturing qualities.

\section{Representation Mode}

While fidelity can often be considered an independent variable of representation, different modes are often coupled with varying levels of fidelity due to the different mediums involved. Research into different representation modes often acknowledges these differences in fidelity, yet focuses on the different representations. Artacho-Ramírez, et al. [18] observed in a case study that the representation mode including, photography, infographic, 3D model, and stereographic 3D model — affected the ability to communicate the product's value to a consumer. Söderman [19] compared representations of cars using sketches, virtual reality, and fully-functional models and noted participant's level of understanding increased slightly with greater realism. Reid, et al. [20] found participants provided inconsistent evaluations and preferences when viewing computer sketches, silhouettes, simple 3D renderings, and realistic 3D renderings. Tovares, et al. [21] surveyed participants with sketches, 3D virtual reality models, and real samples of mugs, finding differences in preference from the representation modes. Macomber and Yang [14], in the same study cited previously, found that 
crowdsourced-responses preferred high-fidelity sketches over CAD renderings. Additionally, their research indicates the importance of considering the amount of time involved in creating different types of representations, suggesting that minimizing the working time for maximal user preference is achieved with quick sketching.

\section{Evaluation of Design Concepts}

Conducting evaluation of design concepts is a critical part of the process for assessing design methods and design tools, as well as relevant to concept selection in a product design process. Evaluation can be performed individually, by a panel of judges, or by aggregating responses, possibly through crowdsourcing. Additionally, those evaluating may be novices or experts in the specific area of design.

Amabile [22] describes creativity as the combination of novelty and appropriateness, and that an idea is creative if independent observers can agree it is creative. Kudrowitz and Wallace [23] developed and tested a metric for evaluating large numbers of ideas at the early stage. Gathering crowd-sourced responses about idea creativity, novelty, and usefulness, they found a positive correlation between creativity and novelty, but insignificant correlation between creativity and usefulness. Tsai [24] studied idea clarity expressed in sketches and found less clearly expressed ideas scored neither highly nor poorly, suggesting participants were unwilling to score ideas they could not fully understand.

\section{Context for New Research}

Prior research has shown how the fidelity and mode of representation can influence perception, and in turn, the evaluation of ideas. Intuitively, we understand that a vast array of design tools is helpful in the design process, and so it is necessary we use these tools because of or despite their varying influence on perception. So far, less attention has been paid to studying how physical prototyping relates to sketching and 3D CAD in the early-stage design process, as well as how all three of these tools perform with regard to the qualities that matter to stakeholders.

This paper draws on the author's doctoral thesis [25] and builds on research published by Häggman, et al. [1]. Starting with the premise that designers are likely aware of certain aspects of how the design tool can influence the design outcome, this research seeks to characterize what designers assume about design tools. The goal is to understand where the separation exists, if any, between what design attributes can be demonstrated to be associated with a design tool, and what design attributes are assumed to be associated.

\section{METHODOLOGY}

\section{Previous Experiment}

This study builds on a previous experiment [1] that was designed to test for differences in quality and quantity of designs produced from sketching, prototyping with blue foam, and modeling in CAD using SolidWorks. Eighteen experienced designers were recruited to individually work on a 2.5-hour design task. In the description of the design prompt, designers were instructed to design a remote control for use with a living room home entertainment center. In total, 83 designs were produced.

All of the designs were re-sketched by a single industrial designer in order to create a consistent presentation, independent of the original design representation (Figure 1). These re-sketched images were then presented to non-design-experts on Amazon's Mechanical Turk platform for feedback about various qualities such as "creativity", "aesthetics", and "intent to purchase".

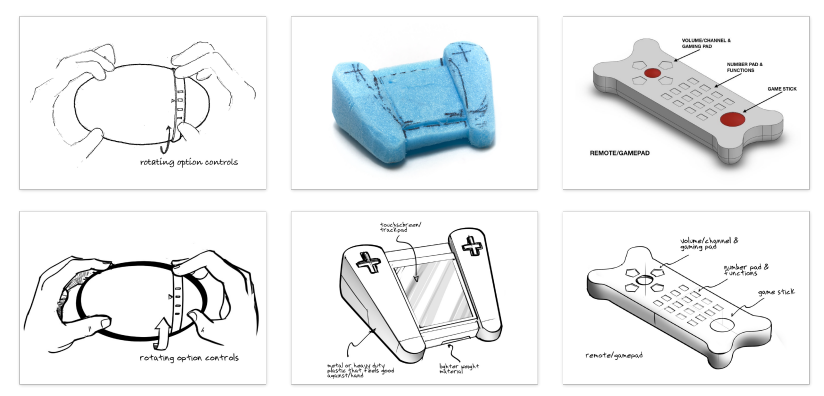

Figure 1 Examples of original designs paired with their re-sketched representations. Sketch is on the left, foam in the middle, and $\mathrm{CAD}$ on the right

\section{Present Survey Design}

The survey was designed to test if people with design experience could identify the original tool by viewing the re-sketch. In its simplest form, this would consist of showing one of the re-sketch images and a multiple-choice question with options for "sketch", "foam", or "CAD".

The survey was constructed as an online survey with three segments. In part one of the survey, participants would be given an explanation of the design and re-sketching process. The first page presented to participants when they enter the survey explains approximately how much time will be required to complete the survey. It describes the survey completion reward: \$20 amazon.com credit for every 10th participant. And it indicates that the participant will receive a score of their performance at the end of the survey.

In part two of the survey, participants would review a set of re-sketch images and make a judgement for each about which design tool was used to create the original. Figure 2 shows the page design for participants to review a re-sketch. 
During the survey, different re-sketch images would be substituted in place using this same page layout. Which re-sketch images were shown to a participant was determined by Adaptive Choice-Based Conjoint, detailed in the next section.

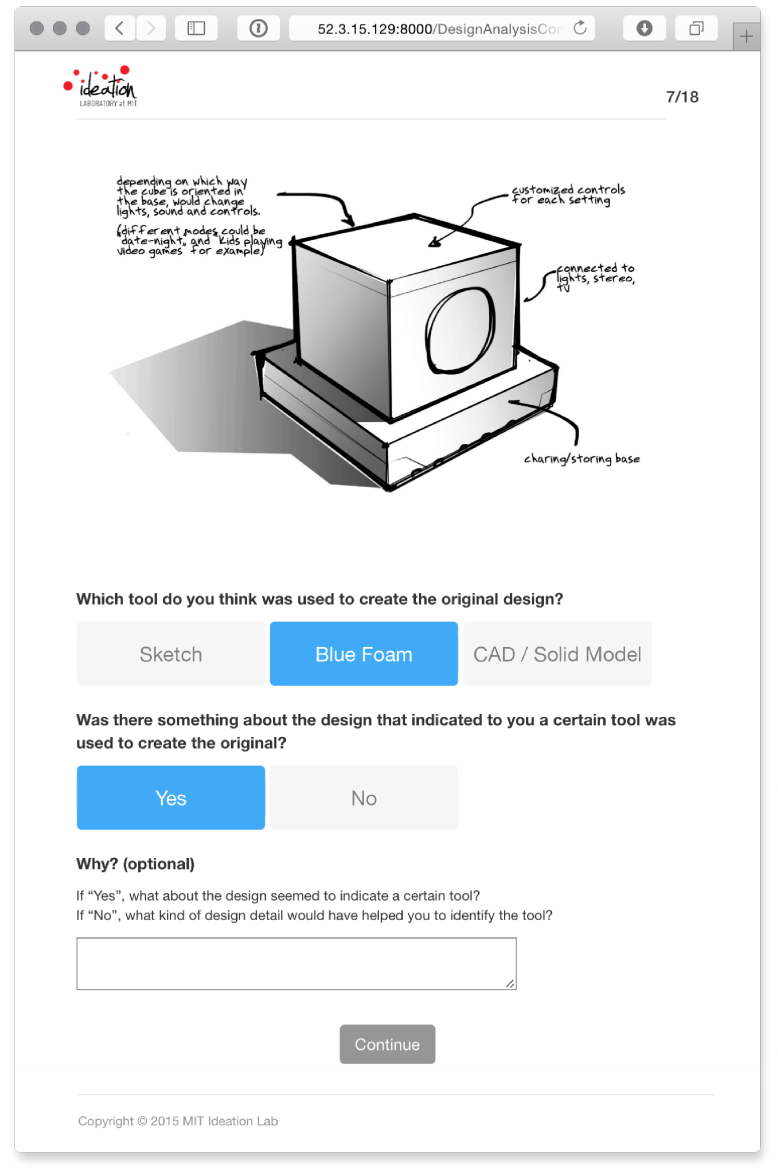

Figure 2 Survey re-sketch review with answers filled

As shown in Figure 2, the first question asked participants to identify the tool that was used to create the original. In the second question, participants were asked to respond "yes" or "no" if something about the design indicated to them a certain tool was used to create the original. Or, in other words, "was this an informed decision?". The third question is a followup to the second, and it asked participants to either identify what about the design indicated a certain tool or to suggest what kind of design detail would have helped to identify the tool.

In part three of the survey, participants would be asked some questions about their experience and general observations. They were asked to indicate their current status as an undergraduate student, graduate student, practitioner / professional, or other; to describe their experience in professional fields such as product designer, mechanical engineer, industrial designer, etc.; and to specify their age range and gender. On the final page of the survey, participants were shown the score of their performance as a percentage. This percentage was calculated from the number of re-sketch images they identified correctly divided by the total.

\section{Using Adaptive Choice-Based Conjoint}

With 83 total re-sketch images needing to be reviewed for this survey, it would not be feasible to have individual participants review all 83 within a single survey. The more images to review, the more data would be collected per participant. Too many images to review, and the participant might feel fatigued or decide not to complete the survey. Several informal pilot studies were conducted in order to determine an ideal number of re-sketch images. From this testing, a set of 18 re-sketch images was chosen as an appropriate number, resulting in a survey that would take about 15-20 minutes from introduction, to reviewing re-sketch images, to completing the exit survey and receiving a score.

In initial designs of the survey, the set of 18 images would be chosen randomly from the total set of 83 images. However, it was expected that recruiting designers and engineers experienced with sketch, blue foam, and CAD would be difficult, and that we would possibly get around 75-200 participants. With 83 total images and a set of only 18 images being reviewed by a single participant, we were interested in a different method that would provide good quality data from a relatively small number of participants.

We chose to use Adaptive Choice-Based Conjoint (ACBC) as the method for choosing which 18 images to display to a participant. While typically used in market research studies, this method is useful for soliciting feedback on a large set of options with a minimal set of questions. This was of interest to us because of the relatively large set of images compared to the number of user responses we were expecting to receive.

In a typical adaptive conjoint study in a market research context, there are at least two phases. Phase one is the self-explicated section. During this phase, the participant indicates her preferences for a series of product attributes. This training section informs the adaptive algorithm of what to present in the next phase.

Phase two is the conjoint section. Here, the participant is shown a series of products, each with different sets of attributes chosen by the adaptive algorithm based on her responses from phase one. Each time she makes a selection of preference, the new data is incorporated into the model in order to improve the efficiency of the conjoint.

Typically, participants of an ACBC survey would choose what they prefer from a set of explicit options. In our survey, the goal is not what participants prefer, but whether they 
think a re-sketch image was originally created with sketch, foam, or CAD. In this way, "sketch, "foam", or "CAD" is the preference. However, without more information about the re-sketch image, we would not know what set of options this shows a preference for.

In order to adapt $\mathrm{ACBC}$ to this type of survey, re-sketch images would need to be tagged with a set of attributes describing the design and its representation. Only the image would be presented to participants of the survey. When a participant made a choice of "sketch, "foam", or "CAD" about a particular image, implicitly she would be asserting a preference for that tool associated with a particular set of attributes. Figure 3 and Figure 4 illustrate this idea with the participant-facing survey on the left and the attributes, normally hidden to the participant, on the right.
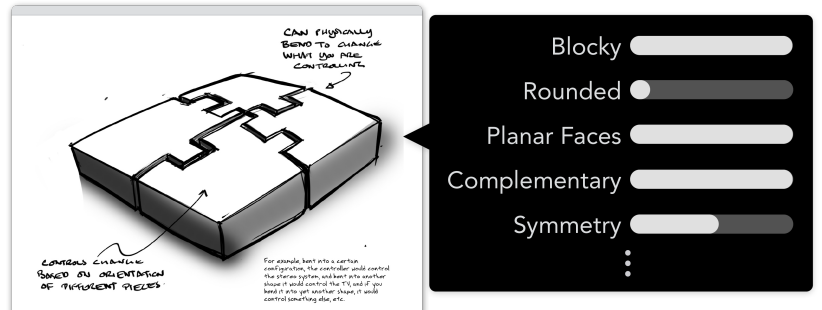

Which tool was used to create the original design?

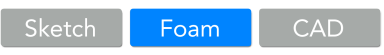

Figure 3 Example re-sketch image with hidden attribute values

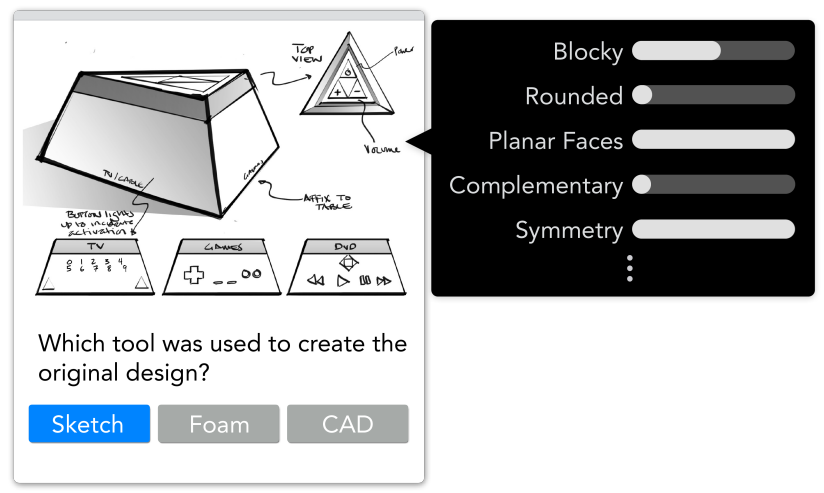

Figure 4 Example re-sketch image with different hidden attribute values

\section{Design Attributes Extended}

From the original research study, we had a set of 15 attributes that described aspects of the design including interaction, form, and input. Input Method describes the physical hardware of the remote that is intended as an affordance for the user to interact with the device. Form Factor describes the physical form of the remote control, what pre-existing form it visually resembles most. Interaction is distinct from Input Method in that it describes the primary type of interaction the user is required to use. While a typical remote may only require hands to press buttons on the remote, another design may require certain body motion to trigger a specific action. This list of attributes and categories is shown in Table 1.

\begin{tabular}{l|l|l} 
Interaction & Form Factor & Input Method \\
\hline hands & standard remote & buttons \\
eyes & game controller & touchscreen \\
body & smartphone & joystick \\
novelty & mouse & scroll wheel \\
& novelty & gestural \\
& & novelty
\end{tabular}

Table 1 Design attributes by category

With the designs already coded with these attributes, we re-reviewed the set of 83 images and took note of any possible detail someone might use to try to determine what tool created the original design. From this review, the authors and an additional graduate researcher extended the set of attributes to include, as examples: blocky, "Does the general form take after a block-like shape?", complexity, "Does the design contain complex geometry or high amounts of detail?", people \& hands, "Does the sketch show people or hands?", wide tolerances, "Does the sketch show wide geometric tolerances, with respect to button sizes/ shapes, imperfect geometric forms?".

In total, we identified 21 additional attributes relating to the idea and its presentation, Table 2. The motivation for these two categories was that the idea, and perhaps artifacts of the design tool present in the idea, or the presentation, and perhaps stylistic choices for how the idea was represented as a sketch, could influence a person's perception of what tool was used to create the original design. We created a survey for all 83 images, and as a research team used a 3-point scale of "Yes", "Neutral", "No" to assign attribute values to each image. The images were presented in a randomized order. The survey was designed and administered using tools from Qualtrics.

Participating in this survey as a panel, we discussed and achieved consensus before assigning a value. These 21 additional attributes (Table 2) were combined with the 15 attributes from the original study to create a 36-attribute description of the design and representation featured in a re-sketched image. 


\begin{tabular}{|c|c|}
\hline Category & Attribute \\
\hline Idea - & $\begin{array}{l}\text { Blocky } \\
\text { Rounded } \\
\text { Planar faces } \\
\text { Complementary } \\
\text { Symmetry } \\
\text { Large scale } \\
\text { Delicate parts } \\
\text { Hinges } \\
\text { Chamfer } \\
\text { Detail out of plane } \\
\text { Complexity } \\
\text { Buttons }\end{array}$ \\
\hline Presentation & $\begin{array}{l}\text { People \& hands } \\
\text { Multiple views } \\
\text { Ground plane } \\
\text { Sketch hairs } \\
\text { 2D appearance } \\
\text { Shading } \\
\text { Stylized text } \\
\text { Wide tolerances } \\
\text { Isometric view }\end{array}$ \\
\hline
\end{tabular}

Table 2 Extended design attributes by category

\section{Survey Flow}

$\mathrm{ACBC}$ requires a self-explicated phase to act as training for the adaptive conjoint. To accomplish this, six re-sketch images were chosen with the goal of spanning a large portion of the attribute space. The six images comprised two examples from each of sketch, foam, and CAD. And for each tool, one image was chosen as the more obvious example of that tool, and one image was chosen as the more non-obvious example of that tool. Figure 5 shows the three "obvious" examples of sketch, foam, and CAD. Figure 6 shows the three "non-obvious" examples.

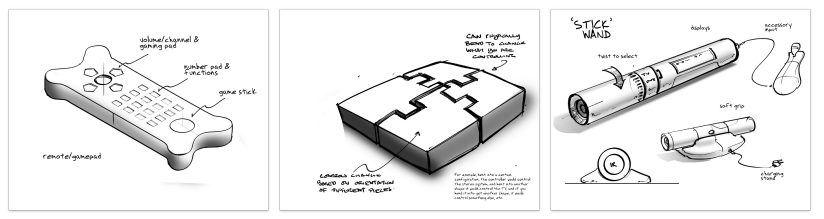

Figure 5 Examples of "obvious" re-sketches

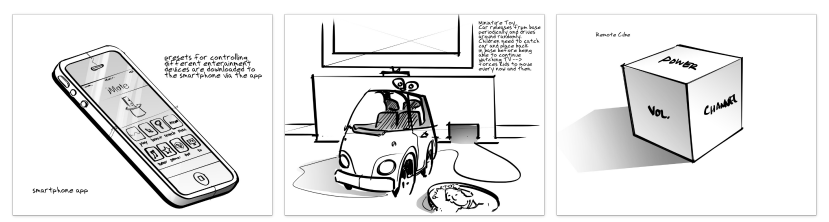

Figure 6 Examples of "non-obvious" re-sketches

For each participant, these same six re-sketch images would be displayed in a random sequence at the start of the survey in order to train the adaptive conjoint. As a consequence of these six images always being displayed to participants, they were reviewed many more times than other images in the set. During the survey, after the six training images were displayed, any 12 of the remaining 77 images would be displayed to the participant. Data from the participant's preferences during the self-explicated section would inform what to display during the conjoint section. This model would continue to be updated during the conjoint section based on the participant's choices.

\section{Recruiting Participants}

Invitations to participate in the survey were typically conducted via email. This includes mechanical engineering product design labs at MIT and undergraduate and graduate courses. We emailed out to product design and consulting companies across the Boston and San Francisco Bay areas. We also emailed out to the IDSA Boston network of professional members and posted to their chapter page on social media.

\section{Rewarding Participants}

The survey introduction told participants that every 10th person would receive a \$20 Amazon.com gift card. This was chosen as a dollar amount that would be appealing enough to respondents, without requiring the budget to pay every individual. In order to maintain a fast return time to respondents, every day new participant entries from the database were assigned a $10 \%$ chance to win.

\section{Demographics}

In total, 108 participants, consisting of undergraduates, graduate students, and professionals, completed the survey. Of the 101 participants who chose to respond, $47 \%$ identified as female and $53 \%$ as male.

Figure 7 shows half of the participants were practitioners and professionals in engineering or design and about one quarter were undergraduates and one quarter were graduate students. A small percentage chose "Other"; based on comments written in the survey, these tended to be people with professional training or experience who were now working in a different field.

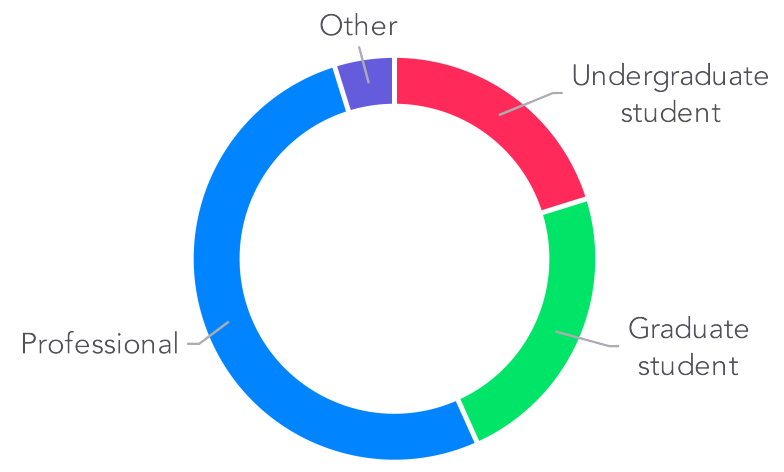

Figure 7 Survey participants' jobs 
Figure 8 shows a histogram of the approximate ages of the participants. With approximately half of the participants as practitioners and professionals and the other half as undergraduates and graduate students, a significant portion of the participants were in the 21-30 year range. The median age was in the 26-30 year range. If we assume a uniform distribution of ages within all of the bins, the average age was 29.4 years.

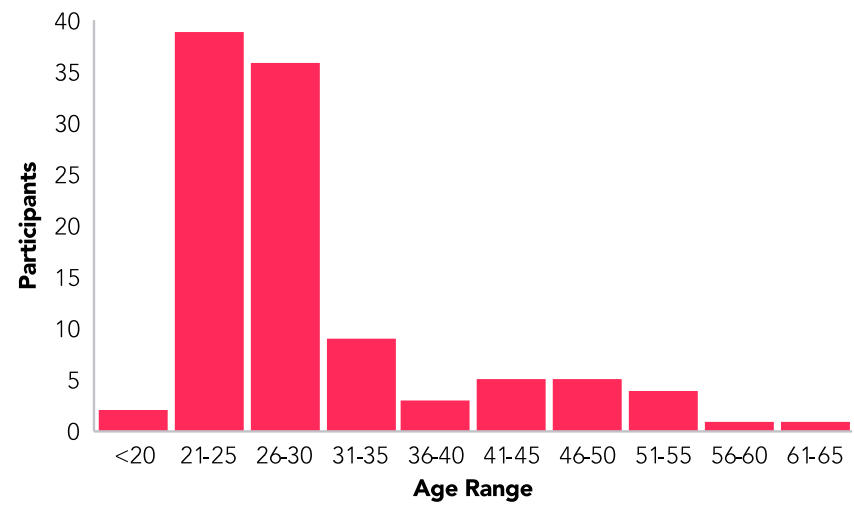

Figure 8 Survey participants' ages

\section{RESULTS}

\section{Accuracy - Whole Population, Students, and Professionals}

Given a 3-choice question, the average accuracy would be $33 \%$ if participants responded purely randomly. If we had observed this result, this would have perhaps indicated there were no discernible differences amongst the re-sketches, even though the designs had begun independently with either sketching, foam prototyping, or CAD. However, we would expect participants to do better than randomly guessing, particularly professionals with significant experience using these tools. In the results from this study, participants correctly identified the original tool used to create the design with $48 \%$ accuracy on average - likely indicating some artifact of the original design tool had been passed along during the re-sketching process.

Participants of this survey ranged from first-year engineering and design students to professionals with decades of experience in industry. How would different levels of experience affect their ability to correctly identify the original tool? Figure 9 shows the participants' averages broken out into groups for "undergraduate students", "graduate students", and "professionals". Recall that "other" was used to describe people with professional training who were currently working in a different field. "Other", as well, represented a small portion of the participants. Therefore, because of their similarities and relative sizes, "professionals" and "other" were combined into one group.

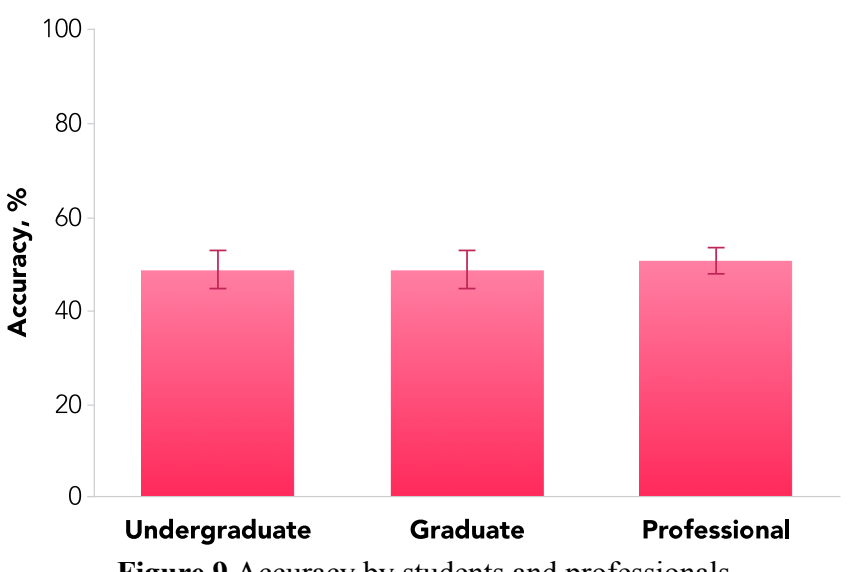

Figure 9 Accuracy by students and professionals

As Figure 9 shows, the differences in accuracy between the three groups is not statistically significant. This would seem to suggest that experience beyond a certain level does not improve an individual's ability to correctly identify the original tool. In future work, it could be informative to re-run the survey with participants who have no prior experience with these tools. Likely, these participants would perform worse than $48 \%$ accuracy, potentially equivalent to random $33 \%$ accuracy.

\section{Accuracy - Sketch, Foam, CAD}

We saw previously that undergraduate students, graduate students, and practitioners performed with highly similar levels of accuracy. What about the different tools: sketch, foam, and CAD? Is one tool easier, or more difficult, to identify accurately? Figure 10 shows accuracy as it is segmented by tool, rather than by experience. When viewed this way, there appears little difference between participants' ability to identify sketch, foam, or CAD.

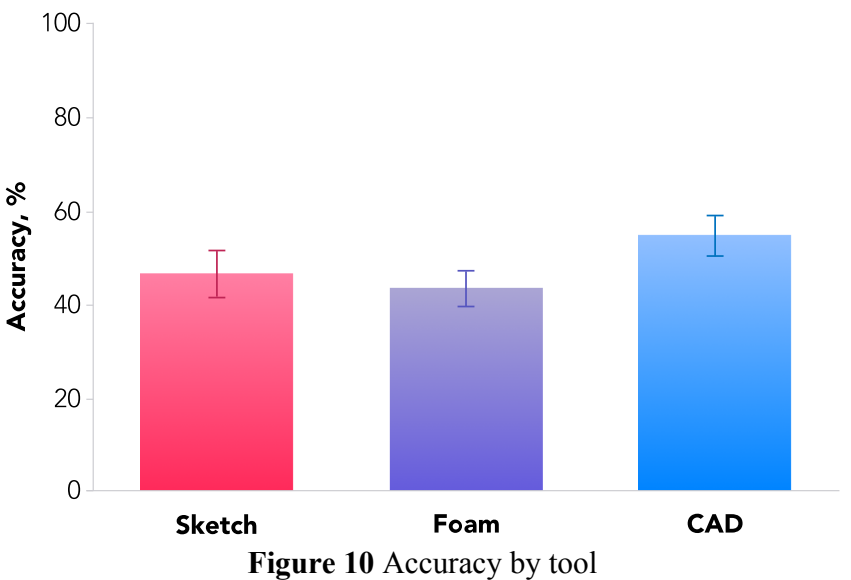

In the survey, each time a participant was presented with an image and asked to identify if it was originally produced via sketch, foam, or CAD, there was a follow-up question about how this decision was informed. In the survey, this 
follow-up question read, "Was there something about the design that indicated to you a certain tool was used to create the original?". Participants were given the option to respond "yes" or "no". Further analysis of the accuracy to identify each tool split the responses for each tool based on whether or not a participant had indicated either "yes" or "no" that "something about the design [indicated] a certain tool was used".

Figure 11 shows this data with "yes/no" responses paired by tool, with the columns on the left of each pair, in more saturated color, representing "yes" and the columns on the right of each pair, in less saturated color, representing "no". Looking at the data this way, it is somewhat surprising to see that accuracies for sketch and CAD do not show a significant difference when split across "yes/no" responses, and that foam does appear to show a significant difference.

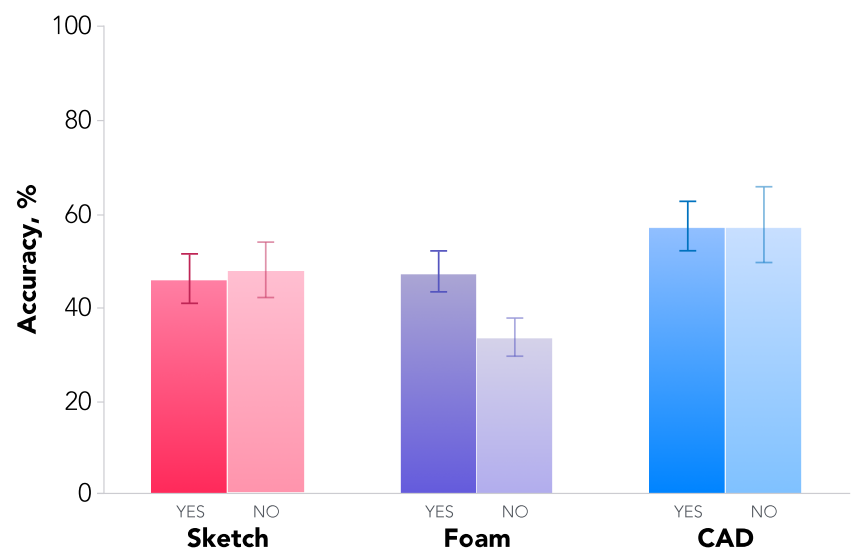

Figure 11 Accuracy by tool with yes/no response

Initially, it was expected that for all three tools accuracy would be higher for "yes" responses and lower for "no" responses. The breakdown for foam does exhibit this, however it is not clear why sketch and CAD appear to show no difference in accuracy between "yes" and "no" responses.

\section{Accuracy - Individual Concepts}

So far, this analysis has looked at the accuracy of participants with different levels of experience and the accuracy of identifying one tool over another. However, what about individual images? Do participants more reliably identify the original tool for some images rather than others? Figure 12 shows all the images presented during the survey from least-accurately identified to most-accurately identified. Lines with more solid color indicate multiple images that were identified with the same accuracy.

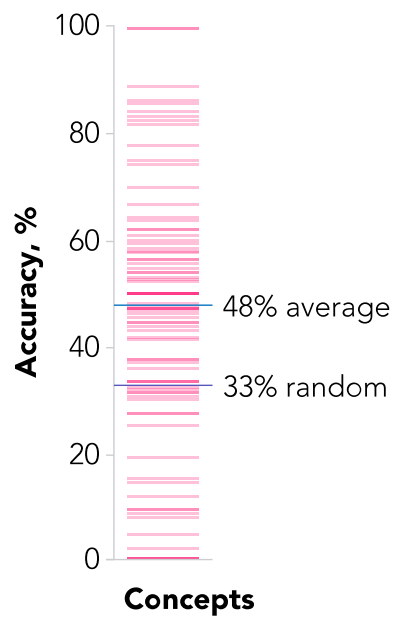

Figure 12 Accuracy of identification of each concept. Lines with more solid color indicate multiple images that were identified with the same accuracy.

The chart shows that the images do vary greatly in terms of what is accurately identified, spanning the entire range from $0-100 \%$. Although the average is that participants correctly identified the original tool with $48 \%$ accuracy, clearly some images were easier to identify than others. What makes these images different?

As an example, image \#74 was correctly identified as a sketch 13/13 times, see Figure 13 which shows both the original sketch (left) and the re-sketch (right). Upon inspection, the image does appear to exhibit certain attributes we might expect of a sketch: looseness of the forms, a roughly two-dimensional appearance, and certain organic and delicate forms (the wires) that might be difficult to produce in foam or CAD.
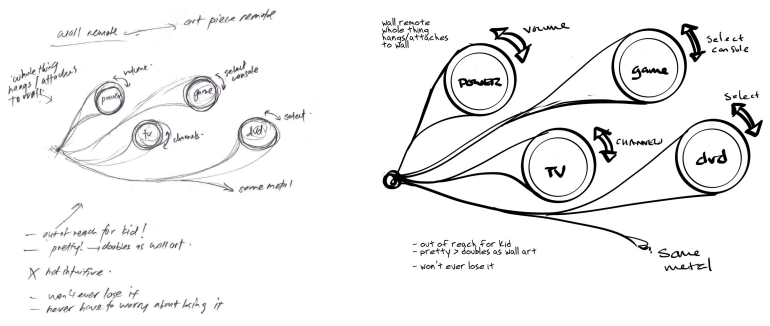

Figure 13 Image \#74: original sketch on the left, re-sketch on the right

In another example, image \#29 was correctly identified as foam only 2/108 times, see Figure 14 (note, this image was always shown during the survey, which is why it was reviewed 108). Upon inspection, this image appears atypical of what one might expect of a foam model. It exhibits a large amount of detail, complexity, and delicate features that might be difficult to produce in foam. Based on this information, the design looks more likely to have been 
produced as a sketch. In fact, 101/106 participants agreed however incorrectly - that this image was a sketch.

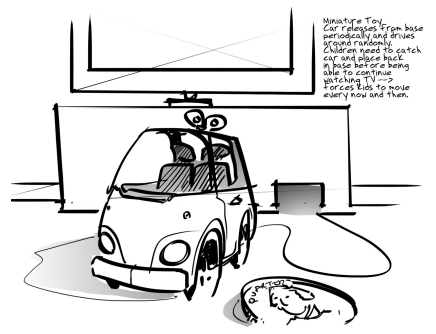

Figure 14 Re-sketch image \#29, originally a foam model

Overall, this suggests that these accuracies are the result of multiple, somewhat independent, factors. One, because participants correctly identified the original tool with $48 \%$ accuracy, some amount of detail from the original design and representation is being carried along into the re-sketch. This gives participants with some degree of familiarity with sketch, foam, and CAD a better-than-random likelihood to identify the original tool.

Two, some designs may be atypical of other designs produced with the same tool. Table 3 goes into detail about what attributes participants associate with a specific design tool, as well as what attributes are associated with the actual tool used in the experiment. However, it is clear that not every design exhibits all the attributes associated with the original tool, nor doesn't exhibit all the attributes not associated with that tool.

Three, some re-sketches are misleading. In the translation process of taking an existing design - whether sketch, foam, or CAD - and creating a new sketch, some amount of interpretation was required on the part of the artist. Inspection of the set of re-sketches and comparing those to their original counterparts showed that three designs exhibited more significant interpretation than others. The best example of this kind of more-significant interpretation can be seen in Figure 15.

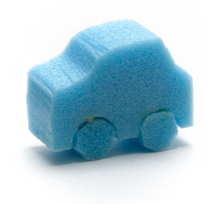

Figure 15 Design \#29, as a foam model
Figure 15 shows the original foam model for comparison with Figure 14. Focusing on the car, you can see the sketch has added significantly more detail to both the exterior and interior of the car. Based on the notes for the foam model, the sketch also added contextual environmental elements including the TV and a coin for scale. As mentioned previously, this image was correctly identified as foam only $2 / 108$ times. Of the set of 83 images, three images, including this one, could be described as receiving more significant interpretation during the re-sketching process. For the two other images, both originally foam prototypes, one was more often mis-identified as a sketch, and the other was mis-identified equally as either a sketch or CAD model.

\section{Quality - Correlation with Surveyed Tool}

In the original study, we used 406 survey responses collected from Amazon Mechanical Turk. Each image received a score for qualities such as "useful", "creative", "comfortable, "intent to purchase", "aesthetics", "clarity of the idea", and "overall better idea". From this data, foam was associated with generating ideas with high creativity, comfort, and aesthetics. CAD was not directly related to low creativity, however it did produce the fewest number of ideas and more ideas was associated with higher creativity.

However, in this study, participants were asked what was the original tool used for creating the design, i.e. what was the surveyed tool. Looking at correlations between the surveyed tool and the qualities data collected previously, there was only one significant correlation ( $p$-value less than 0.05 ), which was the negative correlation between CAD and creativity $(-0.260)$. Previously, this direct association between CAD and low creativity did not exist before in the original study. However, this new result may indicate that participants seem to think CAD produces less creative designs, regardless of whether it actually does.

This distinction between reality and perception of reality what tool was actually used to create the original versus what tool participants chose as the original tool - is important beyond just seeing where there is agreement or disagreement. It can highlight what biases designers and engineers may have about tools and how designs are represented.

\section{Attributes - Correlation with Surveyed Tool and Actual Tool}

In this section, we continue with the idea introduced in the previous section: the distinction between reality and perception of reality. Table 3 shows correlations between attributes of the re-sketch and the surveyed tool, as well as attributes of the re-sketch and the actual tool used. 


\begin{tabular}{|c|c|c|c|c|c|c|}
\hline & Sketch & Foam & CAD & Sketch & Foam & CAD \\
\hline Blocky & -0.254 & 0.247 & 0.045 & -0.383 & 0.396 & -0.005 \\
\hline Rounded & 0.046 & 0.119 & -0.188 & 0.307 & -0.261 & -0.087 \\
\hline Planar faces & & & 0.232 & -0.258 & 0.198 & 0.116 \\
\hline Complementary & -0.156 & 0.292 & & -0.225 & 0.302 & -0.117 \\
\hline Symmetry & & 0.201 & -0.172 & -0.228 & 0.378 & -0.255 \\
\hline Large scale & 0.234 & -0.312 & -0.079 & & 0.019 & -0.153 \\
\hline Delicate parts & 0.084 & -0.212 & 0.204 & 0.128 & -0.244 & 0.204 \\
\hline Hinges & 0.136 & -0.286 & & 0.063 & 0.038 & -0.134 \\
\hline Chamfer & -0.186 & -0.059 & 0.255 & -0.077 & 0.040 & 0.066 \\
\hline Detail out of plane & & -0.058 & -0.066 & 0.363 & -0.328 & -0.049 \\
\hline Complexity & 0.124 & -0.222 & 0.040 & 0.282 & -0.313 & 0.003 \\
\hline Buttons & -0.135 & -0.005 & & 0.143 & -0.337 & 0.265 \\
\hline People \& hands & & 0.464 & -0.384 & -0.159 & 0.395 & -0.395 \\
\hline Multiple views & -0.055 & 0.222 & -0.045 & 0.176 & 0.121 & -0.388 \\
\hline Ground plane & -0.172 & & -0.053 & -0.227 & 0.294 & -0.087 \\
\hline Sketch hairs & & & -0.109 & -0.108 & 0.145 & -0.015 \\
\hline 2D appearance & 0.129 & & & 0.276 & -0.168 & -0.131 \\
\hline Shading & -0.481 & 0.336 & 0.193 & -0.328 & 0.224 & 0.122 \\
\hline Stylized text & 0.085 & -0.001 & -0.069 & 0.097 & 0.000 & -0.126 \\
\hline Wide tolerances & 0.328 & 0.051 & -0.413 & 0.051 & 0.122 & -0.215 \\
\hline Isometric view & -0.030 & -0.085 & 0.258 & -0.209 & 0.147 & 0.171 \\
\hline
\end{tabular}

Table 3 Attributes and tool correlations

In the vertical direction, Table 3 lists 21 of the image attributes related to how the idea was represented as a sketch. The first set of three columns contains Spearman correlations for sketch, foam, and CAD for the surveyed tool (what tool surveyed participants chose as the original tool). The second set of three columns contains correlations for sketch, foam, and CAD for the actual tool used to create the designs.

Cells with bold numbers are correlations with a $p$-value less than 0.05 . One way to read this table is that if participants of the survey were extremely accurate in their choices for what tool was used to create the original, then all of the correlations for surveyed tool would match the correlations for actual tool. Conversely, if survey participants were extremely inaccurate in their choices, then none of surveyed tool correlations would match the actual tool correlations.

Although we are typically interested in where there is a significant correlation, it can be equally interesting where there is no significant correlation. This leads us to a second way to read this table: pick a specific attribute and look at the related surveyed and actual correlations with sketch, foam, and CAD.

For example, pick the attribute "blocky". "Blocky" showed a negative correlation with sketching, a positive correlation with foam, and no significant correlation with CAD amongst the tools participants picked in the survey. By comparison, "blocky" shows a very similar set of correlations with the actual tool used. Interpreting this, designers and engineers tend to associate foam with creating blocky designs, sketching with creating non-blocky designs, and no association of CAD and blocky designs. (This last result of no correlation between $\mathrm{CAD}$ and blockiness is somewhat surprising given our initial assumption that participants would associate CAD with blockiness.) These assumptions, on the part of the surveyed participants, about tools creating design attributes are supported by reality. Given the correlations between actual tool used and blocky design, "blocky" would be productive as an indicator.

As another example, pick the attribute "chamfer". The surveyed tool showed a positive correlation with CAD and no correlation with either sketch or foam. However, this is in contrast to the actual tool which showed no correlation for any of the tools. This suggests that participants assumed a relationship, that chamfers were likely from CAD and not from other tools, when in actuality no such association exists. Or put differently, in the design process, presence or absence of chamfers did not correlate with sketch, foam, or CAD, yet surveyed participants assumed that chamfers were more often from CAD and not from other tools. One possible explanation for why participants made this assumption is the ease with which chamfers are created in CAD - often a matter of a click of a button. 


\section{CONCLUSIONS}

\section{Accuracy}

In the survey, participants demonstrated 48\%, better-than-random accuracy at identifying the original design tool used. This suggests not only that the design tool imparts a design with certain attributes, but also that those attributes often are maintained through the re-sketching process. Segmenting the participants by experience (undergraduate student, graduate student, and professionals) revealed that beyond a minimal level, additional experience did not increase a participant's accuracy.

\section{Quality Correlation with Surveyed Tool}

In the original study, the plurality of top-rated designs for creativity, comfort, and aesthetics came from foam prototypes. In comparison, low creativity correlated with $\mathrm{CAD}$ as the tool chosen during the survey.

\section{Attribute Correlation with Surveyed Tool and Actual Tool}

Correlations between attributes and the surveyed tool showed that participants perceived blockiness as being positively correlated with foam and negatively correlated with sketch. In comparison with the actual tool used to create the original designs, this same positive/negative correlation suggests that participants' assumptions about blockiness and tools is accurate.

Participants' responses showed wide geometric tolerances positively correlated with sketch and negatively correlated with CAD. In this case, participants' assumptions are not supported by reality where there is no significant correlation between wide geometric tolerances and any tool.

\section{Tool and Designer}

For the designer using sketching, foam, and CAD to develop and represent ideas, it's important to consider how the chosen design tool can affect the design outcome. Furthermore, the influence of the design tool on a design concept persists, even when the concept is re-represented as a new sketch. This is relevant for designers who may want to dissociate the idea from the tool that created it.

\section{Research Question}

From prior research, we know that how an idea is represented can influence user's perception. In this paper, we investigated the questions:

Can designers correctly identify the tool used to create a particular design?

What design attributes do designers associate with different design tools?
That designers were able to correctly identify the original design tool provides evidence of the particular effects of a tool on a design. In addition, experience with design tools has informed designers what design attributes to expect to see from the use of a specific tool. This research created a preliminary ontology describing design attributes and how designs can be represented. With this knowledge, we understand that designers may be prejudiced to think CAD is associated with low creativity; that CAD is precise, but sketching is loose; that sketching isn't blocky, but foam is. Actual results from these tools support only some of these biases [1], but these biases are nevertheless relevant to how designers perceive a design.

When setting out to achieve a design goal, designers can do so with the knowledge that the tool they use may both influence the attributes of what they design as well as the perception of the concept. It may not be practical, or effective, to neutralize some of these design-tool/ design-attribute biases, so designers should work with an awareness and respect for what a design representation may communicate beyond the idea itself.

\section{FUTURE WORK}

This research studied what design-tool artifacts remained after the design was translated to a sketch. With this as a guide, further research could take a more active approach and seek to modify a design representation so as to mimic design attributes that would be expected from a different tool. Additionally, this research could be expanded to include a greater variety of design tools. Studying this larger set should help create a better understanding of both the influence of the tool on design outcome and the influence of the representation on design perception.

\section{ACKNOWLEDGEMENTS}

This work was supported in part by the National Science Foundation under the award CMMI-1334267 and by the MIT-SUTD International Design Center. The findings, conclusions, and recommendations expressed are those of the authors and do not necessarily reflect the views of the sponsors.

\section{REFERENCES}

1. Häggman, A., Tsai, G., Elsen, C., Honda, T., \& Yang, M. C. (2015). Connections between the design tool, design attributes, and user preferences in early stage design. Journal of Mechanical Design, 137(7), 071408 .

2. Schon, D. A. (1992). Designing as reflective conversation with the materials of a design situation. Research in Engineering Design, 3(3), 131-147.

3. Acuna, A., \& Sosa, R. (2011). The Complementary Role of Representations in Design Creativity: 
Sketches and Models. In Design Creativity 2010 (pp. 265-270). Springer London.

4. Viswanathan, V. K., \& Linsey, J. S. (2012). Physical models and design thinking: A study of functionality, novelty and variety of ideas. Journal of Mechanical Design, 134(9), 091004.

5. Gerber, E., \& Carroll, M. (2012). The psychological experience of prototyping. Design studies, 33(1), 64-84.

6. Black, A. (1990). Visible planning on paper and on screen: The impact of working medium on decision-making by novice graphic designers.

Behaviour \& Information Technology, 9(4), 283-296.

7. Bilda, Z., \& Demirkan, H. (2003). An insight on designers' sketching activities in traditional versus digital media. Design studies, 24(1), 27-50.

8. Vasantha, G. V. A., Chakrabarti, A., Rout, B. K., \& Corney, J. (2014). Influences of design tools on the original and redesign processes. International Journal of Design Creativity and Innovation, 2(1), 20-50.

9. Mugge, R., \& Schoormans, J. P. (2012). Newer is better! The influence of a novel appearance on the perceived performance quality of products. Journal of Engineering Design, 23(6), 469-484.

10. Pérez Mata, M., Ahmed-Kristensen, S., \& Yanagisawa, H. (2013). Perception of aesthetics in consumer products. In 19th International Conference on Engineering Design (pp. 527-536).

11. McCloud, S. (1993). Understanding comics: The invisible art. Northampton, Mass.

12. Kudrowitz, B., Te, P., \& Wallace, D. (2012). The influence of sketch quality on perception of product-idea creativity. Artificial Intelligence for Engineering Design, Analysis and Manufacturing, 26(03), 267-279.

13. Sauer, J., \& Sonderegger, A. (2009). The influence of prototype fidelity and aesthetics of design in usability tests: Effects on user behaviour, subjective evaluation and emotion. Applied ergonomics, 40(4), 670-677.

14. Macomber, B., and Yang, M. C., 2011, "The Role of Sketch Finish and Style in User Responses to Early Stage Design Concepts," ASME International Design Engineering and Technical Conferences Washington, DC.

15. Viswanathan, V., and Linsey, J., "Design fixation in physical modeling: an investigation on the role of sunk cost," Proc. ASME 2011 International Design Engineering Technical Conferences and Computers and Information in Engineering Conference, American Society of Mechanical Engineers, pp. 119-130.

16. Yang, M. C. (2004, January). An examination of prototyping and design outcome. In ASME 2004 International Design Engineering Technical Conferences and Computers and Information in Engineering Conference (pp. 497-502). American Society of Mechanical Engineers.

17. Hannah, R., Joshi, S., \& Summers, J. D. (2012). A user study of interpretability of engineering design representations. Journal of Engineering Design, 23(6), 443-468.

18. Artacho-Ramirez, M. A., Diego-Mas, J. A., \& Alcaide-Marzal, J. (2008). Influence of the mode of graphical representation on the perception of product aesthetic and emotional features: An exploratory study. International Journal of Industrial Ergonomics, 38(11), 942-952.

19. Söderman, M., 2005, "Virtual reality in product evaluations with potential customers: An exploratory study comparing virtual reality with conventional product representations," Journal of Engineering Design, 16(3), pp. 311-328.

20. Reid, T. N., MacDonald, E. F., \& Du, P. (2013). Impact of product design representation on customer judgment. Journal of Mechanical Design, 135(9), 091008.

21. Tovares, N., Boatwright, P., \& Cagan, J. (2014). Experiential Conjoint Analysis: An Experience-Based Method for Eliciting, Capturing, and Modeling Consumer Preference. Journal of Mechanical Design, 136(10), 101404.

22. Amabile, T., "Social Psychology of Creativity: A Consensual Assessment Technique", Journal of Personality and Social Psychology 43(5), 997-1013 (1982).

23. Kudrowitz, B. M., \& Wallace, D. (2013). Assessing the quality of ideas from prolific, early-stage product ideation. Journal of Engineering Design, 24(2), 120-139.

24. Tsai, G. (2011). Design for Surprise and Idea Generation Methods (Masters thesis). Retrieved from http://hdl.handle.net/1721.1/67804

25. Tsai, G. (2016). The tools we use: a study of user preferences for sketches, prototypes, and CAD models and the influence on design outcome (Doctoral dissertation). Retrieved from https://dspace.mit.edu/handle/1721.1/106785 